The tubes were removed on postoperative day 5 , and he was discharged home the same day.

\section{DISCUSSION}

Chylothorax is a very morbid condition in that high-volume drainage causes respiratory compromise and nutritional and immune deficiency. Conservative management has been recommended for up to 2 weeks before attempting surgery, especially in idiopathic cases, but as minimally invasive techniques have improved, early thoracic duct ligation is associated with faster discharge and better recovery with minimal morbidity. ${ }^{5}$ The approach to the duct has traditionally been through the right side of the chest, even in cases of left chylothorax, because the aorta and esophagus overlie the duct from the left. Traditional surgery also emphasizes mass ligation of the tissues between the aorta and the azygos vein because of difficulty identifying the thoracic duct. The robotic platform allows excellent visualization and meticulous dissection, facilitating a transmediastinal approach and direct identification of the thoracic duct.

The advantage of the left-sided approach in cases of left chylothorax is that inspection of the mediastinum can allow identification of small leaking branches that can be directly addressed. If the chylothorax has been caused by leftsided surgery, approaching the duct from the left avoids morbidity and additional pain on the contralateral side. Finally, the ability to approach the duct from either side gives the surgeon more freedom to perform any necessary concomitant procedures. A robotic technique for left-sided surgery for clipping the supradiaphragmatic thoracic duct can be considered for surgical treatment of left-sided chylothorax.

\section{References}

1. Cope C, Kaiser LR. Management of unremitting chylothorax by percutaneous embolization and blockage of retroperitoneal lymphatic vessels in 42 patients. $J$ Vasc Interv Radiol. 2002;13:1139-48.

2. Christodoulou M, Ris HB, Pezzetta E. Video-assisted right supradiaphragmatic thoracic duct ligation for non-traumatic recurrent chylothorax. Eur J Cardiothorac Surg. 2006;29:810-4

3. Thompson KJ, Kernstine KH, Grannis FW, Mojica P, Falabella A. Treatment of chylothorax by robotic thoracic duct ligation. Ann Thorac Surg. 2008;85: 334-6.

4. Watanabe A, Koyanagi T, Nakashima S, Higami T. Supradiaphagmatic thoracic duct clipping for chylothorax through left-sided video-assisted thoracoscopic surgery. Eur J Cardiothorac Surg. 2007;31:313-4.

5. Paul S, Altorki NK, Port JL, Stiles BM, Lee PC. Surgical management of chylothorax. Thorac Cardiovasc Surg. 2009;57:226-8.

\title{
Recurrent catamenial hemopneumothorax treated by coverage with polyglycolic acid sheets
}

\author{
Mamoru Takahashi, MD, ${ }^{\mathrm{a}}$ Tadashi Matsukura, $\mathrm{MD}, \mathrm{PhD},{ }^{\mathrm{a}}$ Takashi Hirai, $\mathrm{MD}, \mathrm{PhD},{ }^{\mathrm{a}}$ and \\ Nobuya Mino, MD, PhD, ${ }^{\mathrm{b}}$ Fukui and Shizuoka, Japan
}

Catamenial hemopneumothorax is a rare disease entity related to thoracic endometriosis in women of reproductive age, and there is limited surgical experience in terms of treatment. ${ }^{1}$ We successfully treated the disorder using coverage with polyglycolic acid (PGA) sheets.

\section{CLINICAL SUMMARY}

A 36-year-old woman, gravida 0 , with an unremarkable medical history presented at Fukui Red Cross Hospital

\footnotetext{
From the Department of Chest Surgery, ${ }^{a}$ Fukui Red Cross Hospital, Fukui, Japan; and Department of Chest Surgery, ${ }^{b}$ Hamamatsu Rosai Hospital, Shizuoka, Japan.

Disclosures: Authors have nothing to disclose with regard to commercial support.

Received for publication May 8, 2012; revisions received Aug 14, 2012; accepted for publication Aug 23, 2012; available ahead of print Sept 17, 2012.

Address for reprints: Mamoru Takahashi, MD, Fukui Red Cross Hospital, 2-4-1

Tsukimi, Fukui City, Fukui, 918-8501, Japan (E-mail: mt10947@yahoo.co.jp).

J Thorac Cardiovasc Surg 2013;145:300-2

0022-5223/\$36.00

Copyright (C) 2013 by The American Association for Thoracic Surgery

http://dx.doi.org/10.1016/j.jtcvs.2012.08.054
}

(Fukui, Japan) for the investigation of asymptomatic right pleural effusion detected during a routine medical checkup. Thoracic computed tomography revealed extensive pleural effusion on the right side; however, no pneumothorax was identified. Moreover, no bullae or lung nodules were detected. We placed a chest drain in the right side of the chest, and the effusion showed an appearance similar to bloody vaginal discharge. The extent of effusion and air leakage appeared to increase during the onset of menstruation. A high level of cancer antigen 125 (2620 $\mathrm{U} / \mathrm{mL}$ ) was detected in a sample of the pleural effusion fluid. The patient then underwent video-assisted thoracoscopic surgery for precise diagnosis and treatment. Intraoperative findings showed numerous blueberry-like spots on the visceral and parietal pleura and the diaphragm (Figure 1, A). Complete resection of the lesions seemed to be difficult, although biopsy samples were collected during the surgery. Pathologic examination revealed endometrial glands embedded in the visceral pleura, parietal pleura, and diaphragmatic muscles (Figure 1,B). These 


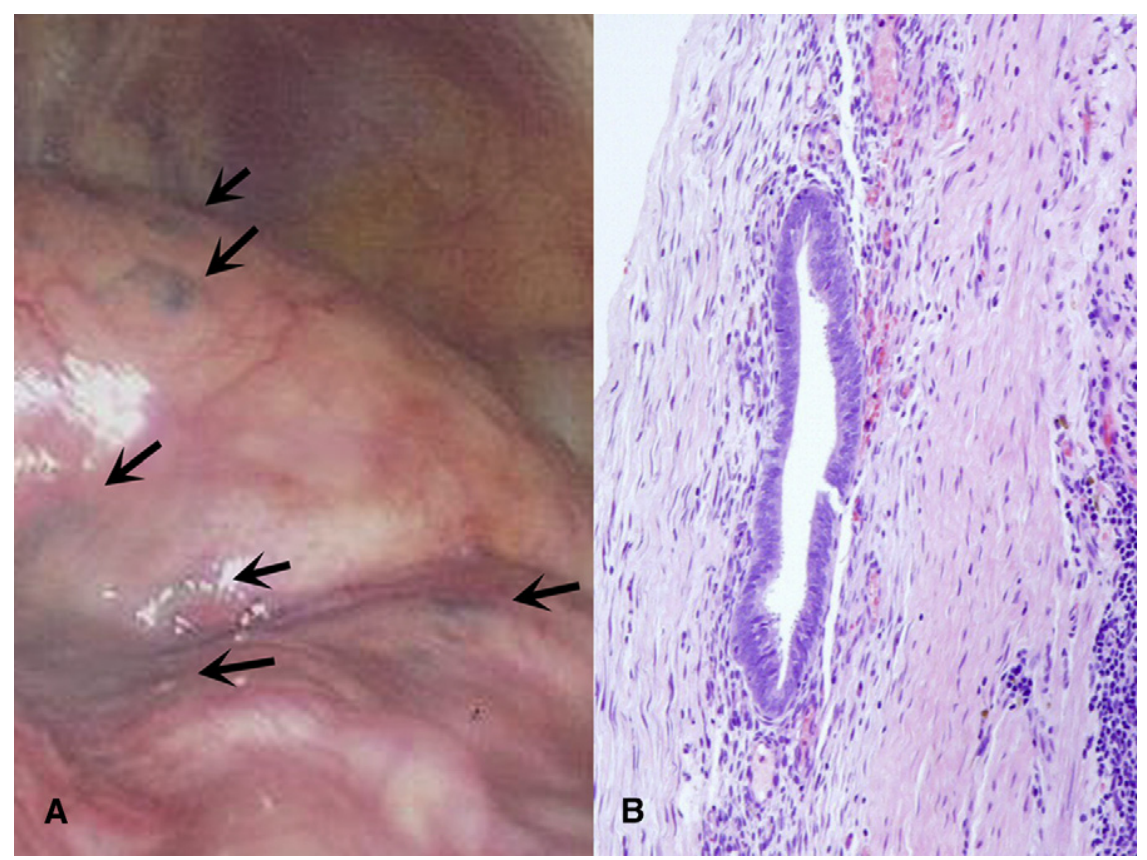

FIGURE 1. A, Intraoperative findings showing endometrial foci (arrows) on the visceral pleura. B, Histopathologic examination reveals an endometrial focus, composed of glands and stroma, implanted in the visceral pleura (hematoxylin-eosin stain; original magnification $\times 100$ ).

findings were compatible with a diagnosis of thoracic endometriosis presenting as catamenial hemopneumothorax. Hormonal therapy with a gonadotropin-releasing hormone analogue was prescribed for the patient; however, she had several occurrences of mild hemopneumothorax. The situation did not change significantly even after switching the medication to a progesterone receptor agonist. Two years later, routine chest radiography revealed an asymptomatic but severe hemopneumothorax. A chemical pleurodesis with OK-432 was performed, but the pneumothorax did not improve; therefore, repeat thoracoscopic surgery was required. The blueberry-like lesions on the visceral pleura had the same widespread pattern observed during the first surgery. While keeping the lung deflated, we covered the entire visceral pleura with tiny, $1-\mathrm{cm}$ square-shaped pieces cut from a PGA sheet (NEOVEIL; Gunze Ltd, Kyoto, Japan) (Figure 2). Complete inflation of the lungs was confirmed before completion of the surgery. The postoperative course was uneventful. The patient stopped the hormonal therapy with a progesterone receptor agonist 1 year after the second surgery because she hoped to become pregnant. Currently, she is doing well with no evidence of relapse 2 years after the second surgery.

\section{DISCUSSION}

This case was unique in the clinical presentation. Thoracic endometriosis mainly shows blueberry-like lesions only on the diaphragm or on one side of the pleura, whereas our patient had numerous lesions on the entire surface of the thoracic cavity. ${ }^{1}$ In addition, the simultaneous occurrence of asymptomatic hemothorax and pneumothorax is rare. ${ }^{1}$ Although the exact pathophysiology of catamenial hemothorax or pneumothorax remains controversial, the pathologic findings in our case suggested a morphologic mechanism as a causative factor, which included sloughing of endometrial tissues in the visceral pleura. $^{2}$

Hemothorax or pneumothorax associated with thoracic endometriosis is often refractory, even to a combination of surgical and chemical approaches. ${ }^{1,2}$ Previous reports have shown the effectiveness of lung-sparing resection in

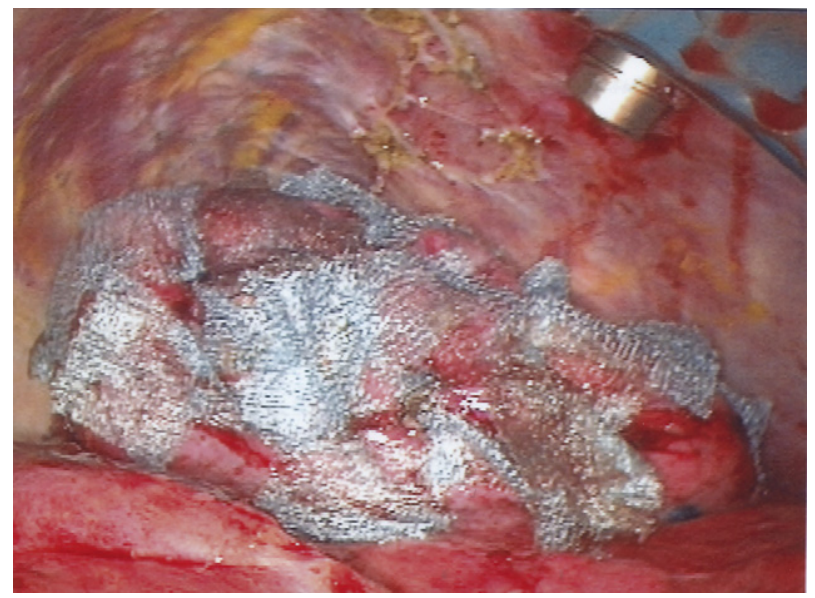

FIGURE 2. Thoracoscopic view showing the lung surface covered with PGA sheets. 
cases in which the endometrial foci are confined to a small area. ${ }^{3}$ However, conventional surgical resection or pleural intervention was not indicated in our case because the endometrial foci were widely spread in the thoracic cavity. Pleural coverage with bioabsorbable materials has been described in a report as total pleural coverage with a regenerative oxidized cellulose mesh. ${ }^{4}$ In the current case, we chose a sheet of PGA, a thin and stretchable bioabsorbable fabric. According to the literature, PGA sheets can be a more useful option for controlling air leakage than regenerative oxidized cellulose mesh because the PGA sheets cause pleural adhesion. ${ }^{4}$ The favorable outcome in this case might suggest that the PGA sheet could be a useful option to seal and strengthen the entire pleura. However, there was a possibility that the pleural adhesions were fostered from other factors, such as pleural scarifications or the preoperative chemical pleurodesis. In addition, the long-term outcome of the PGA sheets for catamenial hemopneumothorax is unknown, and further follow-up is warranted for this case. Although we used only PGA sheets, previous reports have shown that fibrin glues may be an additional option for establishing more firm adhesions. ${ }^{5}$

\section{CONCLUSIONS}

This case of catamenial hemopneumothorax was unique in both clinical presentation and treatment approach. Pleural coverage with PGA sheets may be an option for the treatment of intractable hemopneumothorax.

\section{References}

1. Alifano M, Trisolini R, Cancellieri A, Regnard JF. Thoracic endometriosis: current knowledge. Ann Thorac Surg. 2006;81:761-9.

2. Korom S, Canyurt H, Missbach A, Schneiter D, Kurrer MO, Haller U, et al. Catamenial pneumothorax revisited: clinical approach and systemic review of the literature. J Thorac Cardiovasc Surg. 2004;128:502-8.

3. Ziedalski TM, Sankaranarayanan V, Chitkara RK. Thoracic endometriosis: a case report and literature review. J Thorac Cardiovasc Surg. 2004;127:1513-4.

4. Noda M, Okada Y, Maeda S, Sado T, Sakurada A, Hoshikawa Y, et al. An experience with modified total pleural covering technique in a patient with bilateral intractable pneumothorax secondary to lymphangioleiomyomatosis. Ann Thorac Cardiovasc Surg. 2010;16:439-41.

5. Ueda K, Tanaka T, Li TS, Tanaka N, Hamano K. Sutureless pneumostasis using bioabsorbable mesh and glue during major lung resection for cancer: who are the best candidates? J Thorac Cardiovasc Surg. 2010;139:600-5. 